\title{
Testing eigenstate decoherence hypothesis in a model of collisional decoherence
}

\author{
Ivan V. Dudinets ${ }^{1,2}$, Igor Ermakov ${ }^{1,2,3}$, Oleg Lychkovskiy ${ }^{1,2,3}$ \\ ${ }^{1}$ Skolkovo Institute of Science and Technology, Skolkovo Innovation Center 3, Moscow 143026, Russia \\ ${ }^{2}$ Moscow Institute of Physics and Technology, Institutsky per. 9, Dolgoprudny, Moscow region, 141700, Russia and \\ ${ }^{3}$ Department of Mathematical Methods for Quantum Technologies, \\ Steklov Mathematical Institute of Russian Academy of Sciences, Gubkina str. 8, Moscow 119991, Russia
}

(Dated: November 30, 2020)

\begin{abstract}
The eigenstate decoherence hypothesis (EDH) asserts that each individual eigenstate of a large closed system is locally classical-like. We test this hypothesis for a heavy particle interacting with a gas of light particles. This system is paradigmatic for studies of the quantum-to-classical transition: The reduced state of the heavy particle is widely believed to rapidly loose any nonclassical features due to the interaction with the gas. Yet, we find numerical evidence that the EDH is violated: certain eigenstates of this model are manifestly non-classical. Only the weak version of EDH referring to the majority (instead of the totality) of eigenstates holds.
\end{abstract}

Introduction. Quantum mechanical state space is dramatically different from the classical state space. For example, a state of a point particle in classical mechanics is completely determined by its coordinate and momentum, and thus the state space is the two-dimensional phase space. In quantum mechanics the coordinate and momentum can not be determined simultaneously. However, the superposition principle of quantum theory legitimizes arbitrary superpositions of different coordinates (or momenta). As a result, the state space becomes an infinite-dimensional projective Hilbert space. Most of the points in the Hilbert space represent weird superpositions that do not make any sense from the classical point of view. These weird states apparently do not emerge in our everyday experience (and for this reason do not enter the classical formalism). Explaining why this happens is the key for understanding the quantum-toclassical transition.

A conceptually straightforward explanation is provided by the decoherence theory [1-6]. One observes that the weird superposition states turn out to be extremely fragile when the interaction between the system and its environment is taken into account. To demonstrate this fact one often considers a system $\mathcal{S}$ (e.g. a point particle) and its environment (bath) $\mathcal{B}$ (e.g. the gas the particle is immersed in) as a combined closed systems $\mathcal{H}$, whose state $\Psi_{t}$ evolves according to the Schrödinger equation $i \partial_{t} \Psi(t)=H \Psi(t)$, $H$ being the total Hamiltonian that describes the system, the environment and their interaction (we adopt the convention $\hbar=1$ ). The state of the (now open) system $\mathcal{S}$ is described by the reduced density matrix

$$
\rho^{\mathcal{S}}(t) \equiv \operatorname{tr}_{\mathcal{B}}|\Psi(t)\rangle\langle\Psi(t)|,
$$

where $\operatorname{tr}_{\mathcal{B}}$ is the partial trace over the environment $\mathcal{B}$. It has been verified in various physical settings that even when $\rho^{\mathcal{S}}(0)$ is a projection on a highly non-classical state, $\rho^{\mathcal{S}}(t)$ becomes a mixture of classical-like states on a certain $d e$ coherence time scale, the latter being extremely short for a system $\mathcal{S}$ that can be regarded as macroscopic (e.g. for a macroscopic point particle) [6].
Importantly, the focus of the above-described studies in the framework of the decoherence theory has always been on out-of-equilibrium initial states $\Psi(0)$. In particular, often $\Psi(0)$ of the product form has been considered, describing initially uncorrelated system and environment. A complementary view can be provided by considering the eigenstates of the combined system $\mathcal{H}$. They remain unchanged under the Schrödinger equation (apart from the irrelevant phase factor), and one naturally expects that they are locally classical-like from the outset. This is the essence of the eigenstate decoherence hypothesis $(\mathrm{EDH})$ put forward by one of us some time ago [7]. More formally, the EDH asserts that for any eigenstate $\Phi_{E}$ of the total Hamiltonian $H$ the corresponding reduced density matrix

$$
\rho_{E}^{\mathcal{S}} \equiv \operatorname{tr}_{\mathcal{B}}\left|\Phi_{E}\right\rangle\left\langle\Phi_{E}\right|
$$

is classical-like according to a suitable quantumness measure, or, simply put, free of any weird quantum Schrödinger-cat-type superpositions.

The EDH is motivated by the eigenstate thermalization hypothesis $(\mathrm{ETH})[8-10]$. The later asserts that $\rho_{E}^{\mathcal{S}}$ is a smooth function of $E$. This implies, by standard statistical arguments, that $\rho_{E}^{\mathcal{S}}$ is thermal, and that a long-time average of any evolving state with subextensive energy uncertainty is thermal [11]. Various case studies have suggested that $\rho_{E}^{\mathcal{S}}$ indeed has all attributes of an equilibrium state for generic nonintegrable systems without disorder [10, 12, 13]. On the other hand, it has been recently realized that many nonintegrable systems possess quantum many-body scars, i.e. abnormal states in the bulk of the otherwise thermal spectrum that violate the ETH [14-16].

One reasonably expects that if a state is thermal, the more so it should be classical-like [5]. Combining this straightforward idea with the ETH, one immediately arrives to the EDH [7]. Furthermore, one can expect that the EDH should be valid for a larger class of systems than the ETH, e.g. for interacting integrable systems [7] and system with quantum many-body scars. This expectation is rooted in the fact that integrability or presence of scars is a global property sensitive e.g. to long range interactions, 
and a system is not able to explore whether it is integrable, scarred or fully ergodic on an extremely short decoherence time scale.

The EDH has not been tested in specific models up to now, with the exception of a central spin model [7]. Here we fill this gap by testing the EDH for a paradigmatic case of a heavy particle (subsystem $\mathcal{S}$ ) interacting with a gas of light particles (environment $\mathcal{B}$ ). As established in the early days of the decoherence theory [5, 17, 18] and elaborated later on [19-23], a non-equilibrium spatially nonlocal state of a heavy particle rapidly turns into a mixture of well-localized classical-like states due to collisions with the particles of the gas (quantitative predictions of this theory were verified in interference experiments with fullerenes [24]). Yet, surprisingly, we find that there exist non-classical eigenstates of the combined system $\mathcal{H}$ with a coherence length on the order of the system size.

The rest of the paper is organised as follows. First we introduce the model. Then we introduce a figure of merit of the (non-)classicality - the coherence length, and discuss how it can be used to test the EDH. Then we consider a trivially integrable case of an infinitely heavy particle. We demonstrate that in this case the EDH can be either valid or dramatically violated, depending on the spatial symmetry of the system. Next, we present the results of a numerical studies of a nonitegrable system. They strongly indicate the violation of the EDH. We conclude the paper by discussing the implications of this finding.

Model. We study a closed quantum system consisting of a distinguished particle (referred to simply as "the particle" in what follows) interacting with the Fermi gas, the latter being regarded as an environment for the former. For the sake of numerical calculations, we consider a onedimensional lattice variant of this system with $N$ fermions and a single particle, all hopping on a linear lattice with $L$ sites. The Hamiltonian reads

$$
\begin{aligned}
H= & \left(-J \sum_{i=1}^{L-1} c_{i}^{\dagger} c_{i+1}-J^{\prime} \sum_{i=1}^{L-1} a_{i}^{\dagger} a_{i+1}+\text { h.c. }\right) \\
& +\sum_{i=1}^{L} U a_{i}^{\dagger} a_{i} c_{i}^{\dagger} c_{i}+\delta H .
\end{aligned}
$$

Here $c_{i}^{\dagger}$ creates a fermion and $a_{i}^{\dagger}-$ the particle, $J>0$ and $J^{\prime} \geq 0$ are hopping constants for fermions and the particle, respectively, $U>0$ is the coupling constant between a fermion and the particle. The term

$$
\delta H=\epsilon \sum_{j=1}^{L}(j / L) c_{j}^{\dagger} c_{j}
$$

describes the linear on-site potential felt by fermions. It is introduced to break the otherwise present reflection symmetry of the Hamiltonian, for a purpose to be discussed later. When considering large system size, we will imply the thermodynamic limit with the fixed fermionic density $n \equiv N / L$.
In order to separate time scales of decoherence and thermalization, we assume that the distinguished particle is heavy as compared to the fermions [21, 25], which amounts to $J \gg J^{\prime}$.

In the limiting case of the infinitely heavy particle, $J^{\prime}=$ 0 , the model becomes trivially integrable, with the eigenstates of the form

$$
\Phi_{E}=|j\rangle \otimes\left|F_{E}^{j}\right\rangle
$$

where $|j\rangle$ is the state of the particle localized on the site $j$ and $\left|F_{E}^{j}\right\rangle$ is an eigenstate of the $j$-dependent quadratic fermionic Hamiltonian

$$
H_{j}=\left(-J \sum_{i=1}^{L-1} c_{i}^{\dagger} c_{i+1}+h . c .\right)+U c_{j}^{\dagger} c_{j}+\delta H .
$$

Note that if $\delta H=0$, the spectrum acquires degeneracies due to the reflection symmetry of the Hamiltonian.

Coherence length and $\mathrm{EDH}$. In order to formulate the $\mathrm{EDH}$ for a specific subsystem $\mathcal{S}$, one needs to define a suitable measure of its quantumness. To this end we employ a natural measure of quantumness of the state $\rho^{\mathcal{S}}$ of a point particle - its coherence length [26, 27],

$$
l\left(\rho^{\mathcal{S}}\right) \equiv \sqrt{\frac{2 \sum_{i, j=1}^{L}\left|\left\langle i\left|\rho^{\mathcal{S}}\right| j\right\rangle\right|^{2}(j-i)^{2}}{\sum_{i, j=1}^{L}\left|\left\langle i\left|\rho^{\mathcal{S}}\right| j\right\rangle\right|^{2}}} .
$$

Here $\rho^{\mathcal{S}}=\operatorname{tr}_{\mathcal{B}}|\Psi\rangle\langle\Psi|$ is a reduced density matrix of the particle obtained from a pure state $\Psi$ of the closed particlegas system and $\left\langle i\left|\rho^{\mathcal{S}}\right| j\right\rangle=\left\langle\Psi\left|a_{j}^{\dagger} a_{i}\right| \Psi\right\rangle$ is its matrix elements in the position basis 1 The coherence length effectively measures the spatial extension of a superposition of localized states, ranging from 0 for a particle localized on a single site to $(L-1)$ for a highly non-classical state of the form

$$
\Psi_{Q}=(1 / \sqrt{2})\left(a_{1}^{\dagger}+a_{L}^{\dagger}\right)|F\rangle
$$

where $|F\rangle$ is some state of $N$ fermions.

To simplify the terminology and notations, we will attribute coherence length also to pure many-body states, implying $l(\Psi) \equiv l\left(\operatorname{tr}_{\mathcal{B}}|\Psi\rangle\langle\Psi|\right)$.

Importantly, while $l$ for nonclassical states can be on the order of the size of the system $L$, as in eq. [8), for classicallike states $l$ is independent on the system size. In particular, for the model (3) we expect that $l$ for classical-like states is bounded from above by the average interparticle distance $L / N$ times the probability for the scattering of a fermion off the particle. This expectation is grounded in understanding that each scattering event effectively entangles the heavy particle with the fermionic environment and thus causes decoherence. Note that this physical picture implies a non-equilibrium process and may not apply to

\footnotetext{
${ }^{1}$ Note that our definition (7) is different from that in [26] by a factor $\sqrt{2}$.
} 
equilibrium states. We emphasize that the hallmark of the EDH would be not some specific value of the coherence length of eigenstates accepted as classical (this is a matter of convention), but independence of the coherence length on the system size in the thermodynamic limit. The latter property is in the focus of the present study.

Analogously to the ETH [11], one can discriminate between strong and weak forms of the EDH. The former concept implies that the EDH is valid for all eigenstates. In order to confirm the strong the EDH in our model, one needs to verify that the maximum over all eigenstates of the coherence length, $l_{\max }$, stays bounded when the system size grows. The weak EDH stands for a somewhat more vague conjecture that a vast majority of eigenstates satisfy the EDH. We will regard the weak EDH satisfied whenever the coherence length $l_{\text {av }}$ averaged over all eigenstates remains bounded with the system size increasing.

EDH: integrable case. In the integrable case, $J^{\prime}=0$, each eigenstate (5) has zero coherence length and EDH is perfectly valid. However, in the case $\delta H=0$ the symmetry of the Hamiltonian leads to a caveat: all eigenstates of the form (5) are doubly degenerate, and one can construct new eigenstates with a large coherence length. Consider e.g an eigenstate

$$
\widetilde{\Phi}_{E}=\frac{1}{\sqrt{2}}\left(|1\rangle \otimes\left|F_{E}^{1}\right\rangle+|L\rangle \otimes\left|F_{E}^{L}\right\rangle\right),
$$

where $\left|F_{E}^{1}\right\rangle$ and $\left|F_{E}^{L}\right\rangle$ are eigenstates of fermionic Hamiltonians $H_{1}$ and $H_{L}$, respectively. Its coherence length reads

$$
l\left(\widetilde{\Phi}_{E}\right)=(L-1)\left|\left\langle F_{E}^{1} \mid F_{E}^{L}\right\rangle\right|^{2} / \sqrt{1+\left|\left\langle F_{E}^{1} \mid F_{E}^{L}\right\rangle\right|^{2}} .
$$

The overlap $\left|\left\langle F_{E}^{1} \mid F_{E}^{L}\right\rangle\right|^{2}$ entering this formula vanishes exponentially in the system size in the thermodynamic limit for most of the energy levels. For this reason the weak EDH is still satisfied. However, for some energy levels, in particular, for those corresponding to ground states of $H_{1}$ and $H_{L}$, the overlap vanishes as slow as $1 / L^{\alpha}$, which is a manifestation of the Anderson orthogonality catastrophe [28]. Importantly, the exponent $\alpha$ does not exceed unity [29]. As a consequence,

$$
l\left(\widetilde{\Phi}_{E}\right) \sim L^{1-\alpha}, \quad \alpha<1
$$

diverges in the thermodynamic limit. Thus we come to the conclusion that for an integrable Hamiltonian with a degenerate spectrum some eigenstates have the coherence length on the order of the size of the system and thus violate the strong EDH spectacularly $\left.\right|^{2}$ This conclusion is

\footnotetext{
2 There is a very different type of anomalous, highly excited eigenstates that violate the strong EDH. Namely, one can construct a many-body eigenstate of the free Fermion gas with zero densities at two distinct sites; the tensor product of this state and a superposition of the particle over these two sites is then an eigenstate of the entire Hamiltonian (3) with $J^{\prime}=0$ and $\delta H=$ 0 . These states are specific for point interaction on a lattice and do not emerge for finite-range interactions, in continuum models or for finite mass of the particle, therefore we do not consider them in detail.
}

particularly stunning in light of the fact that the integrable model under considerations is often employed in textbook demonstrations of the collisional decoherence [6]. However, in these demonstrations the decoherence process is viewed as a far-from-equilibrium dynamics described by a sequence of pairwise collisions of gas particles with the impurity particle. It is not very surprising that this approach may be inapplicable to equilibrium states in the bottom of the spectrum.

If one breaks the reflection symmetry of the Hamiltonian (3) by a nonzero $\delta H$, the degeneracy of the spectrum goes away, the construction (9) becomes impossible and no eigenstates with a nonzero coherence length emerge. The strong EDH is satisfied in this case.

Curiously, the Hamiltonian (3) with $\delta H=0$ has another integrable point, $J=J^{\prime}$ [30, 31]. This case is superficial for studying the quantum-to-classical transition, since there is no separation of time scales of decoherence and thermalization, and there is no reason to expect the particle to be "more classical" than its environment. However, from the technical point of view, it would be instructive to check whether the coherence lengthes of some of eigenstates diverge for this another integrable point of the model. However, the explicit calculations in this model constitute a formidable challenge. Up to now, the only available analytical result is the particle's density matrix for the ground state in a translation-invariant system at a fixed total momentum, that implies a finite coherence length for this state [32].

EDH: nonintegrable case. To test the EDH in the nonintegrable case we numerically diagonalize the Hamiltonian (3) for finite system sizes and calculate the coherence length $l\left(\Phi_{E}\right)$ for each eigenstate $\Phi_{E}$. The range of system sizes is $L=8,9, \ldots 12$. To keep the fermion density $N / L$ as independent on the system size as possible, we choose to half-fill the chain with fermions. Namely, we take $N=L / 2$ for even $L$ and $N=(L-1) / 2$ for odd $L$. We consider both unbiased $(\varepsilon=0)$ and biased $(\varepsilon=0.1)$ versions of the Hamiltonian (3). Note that in the former case, despite the symmetry, the spectrum is nondegenerate.

The coherence lengthes of all eigenstates are presented in Fig. 11 Most of the states have coherence length on the order of the average interparticle distance, $L / N \simeq 2$, in accordance with expectation discussed above. In particular, the coherence length averaged over all states reads $l_{\text {av }}=2.63$. However, one can clearly see that there exists a number of "outlier" eigenstates with a large coherence length well exceeding this value. Such outliers are mostly concentrated at the edges of the spectrum, however they also can be found in the middle of the spectrum.

As discussed above, a decisive signature of violation of the EDH is the growth of the coherence length with the system size. In Fig. 2 we show the finite-size scaling of the average and maximal (over all eigenstates) coherence lengths. The scaling of these two quantities is drastically 


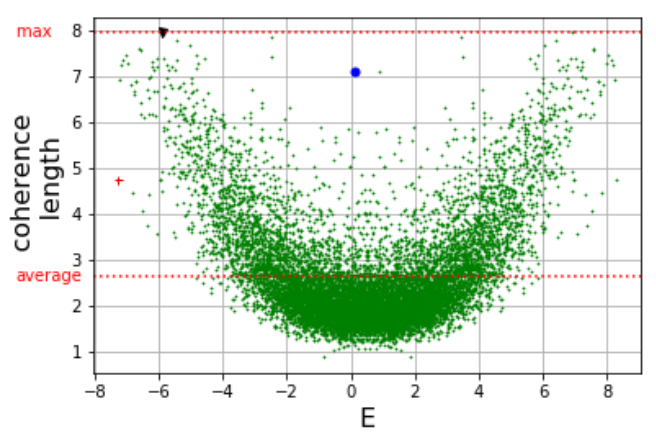

FIG. 1. The coherence length for eigenstates of the nonintegrable Hamiltonian (3) with $J=1, J^{\prime}=0.2, U=1, N=6, L=$ $12, \delta H=0$. The ground state, the state with the maximal coherence length and a state in the middle of the spectrum with a large coherence length are marked with the red cross, black triangle and blue circle, respectively. The correlation function for these three states is shown in Fig. 3

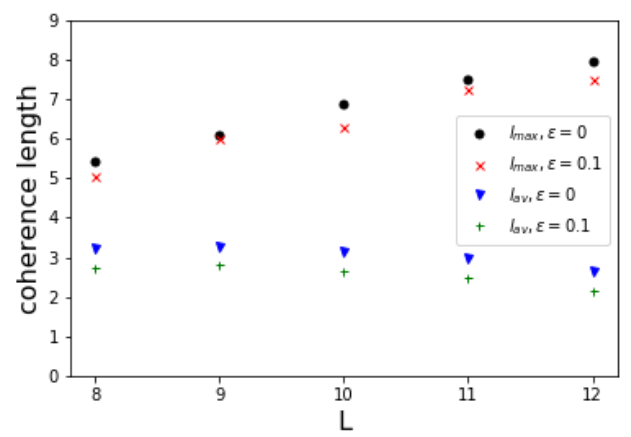

FIG. 2. The maximal $l_{\max }$ and average $l_{\text {av }}$ coherence lengths for eigenstates of the nonintegrable Hamiltonian for the cases of the presence $(\epsilon=0)$ and absence $(\epsilon=0.1)$ of the spatial reflection symmetry. The system is half-filled with fermions, with $N=$ $L / 2$ for even $L$ and $N=(L-1) / 2$ for odd $L$. Other parameters of the Hamiltonian are the same as in Fig. 1 .

different: while the former vanishes with the system size, the latter grows linearly with $L$. Thus we conclude that our numerical data support the weak EDH, but suggest the violation of the strong EDH.

We have performed numerical calculations with various fermion densities (where we can access slightly larger system sizes, up to $L=12$ ), coupling strengths, as well as next-to-nearest neighbour interactions, and obtained similar results. Note that, in contrast to the integrable case, the potential bias $\delta H$ does not quantitatively alter the result, as is clear from Fig. 2

To highlight the difference of outliers from typical eigenstates, we plot in Fig. 3 an absolute value of a correlation function $\left\langle\Phi_{E}\left|a_{j}^{\dagger} a_{1}\right| \Phi_{E}\right\rangle$ (as a function of the position $j$ ) for several outliers and compare it to the same quantity averaged over all eigenstates. One can see that while the av-

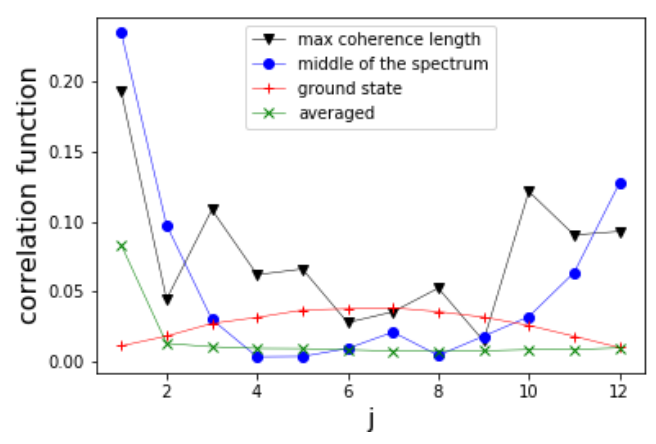

FIG. 3. Absolute value $\left|\left\langle\Phi_{E}\left|a_{j}^{\dagger} a_{1}\right| \Phi_{E}\right\rangle\right|$ of a correlation function of a particle for three outlier eigenstates (highlighted in Fig. 1), compared to the same quantity averaged over all eigenstates. While the latter quantity rapidly decays at the length scale equal to the average distance between the fermions, the correlations for the outliers extend over the entire system. The parameters of the Hamiltonian are the same as in Fig. 1

eraged correlation function demonstrates a rapid decay at the length scale $L / N$, the correlation function of outliers extends over the entire system, even up to the opposite end of the chain.

Summary and discussion. To summarise, we have tested the eigenstate decoherence hypothesis (EDH) for a system consisting of a heavy particle immersed in a onedimensional Fermi gas. We have addressed the integrable case of an infinitely heavy particle as well as the nonintegrable case of a particle of a finite mass. In the integrable case the EDH (both strong and weak versions) can be violated when the spatial symmetries of the model impose a massive degeneracy of the spectrum. On the other hand, if this degeneracy is lifted by a symmetry-breaking potential (integrability being preserved), the strong EDH is valid.

The most surprising results have been obtained numerically for a nonintegrable case. Our numerical data suggest that while the weak EDH holds, the strong EDH is violated by rare nonclassical outlier eigenstates with a particle coherence length on the order of the system size.

The existence of such outlier eigenstates is in stark contrast with the intuition based on the theory of collisional decoherence [5, 17,-21], which predicts a rapid decoherence as soon as the particle experience a collision with the gas particles. A plausible resolution of this conundrum is that most initial states (in particular, product states of a particle and a gas often considered in this theory) have a vanishing overlap with outliers, and thus the latter typically have no effect on the decoherence. A careful crafting of the state of a many-body system (e.g. in a cold atom simulator [33]) is required to unveil the nonclassical eigenstates.

Finally, we remark that the outlier nonclassical eigenstates violating the EDH resemble the many-body scars violating the ETH. One may wonder if the scars, in fact, exist in the model (3). At $J=J^{\prime}$ and $\delta H=0$ the Hamilto- 
nian (3) is a Hubbard Hamiltonian, where scars do exist [34-36]. However, to the best of our knowledge, no scars have been reported away from this point. Furthermore, typically the scars are not robust with respect to perturbations of the Hamiltonian [37], while the violation of the EDH is. We therefore conclude that, anyway, the violation of EDH is not conditioned on the existence of quantum many-body scars.

Acknowledgements. OL is grateful to Oleksandr Gamayun and Anatoly Dymarsky for useful discussions. This work is supported by the Russian Foundation for Basic Research under grant 18-32-20218.

[1] H.D. Zeh, "On the interpretation of measurement in quantum theory," Foundations of Physics 1, 69-76 (1970).

[2] H.D. Zeh, "Toward a quantum theory of observation," Foundations of Physics 3, 109-116 (1973).

[3] W.H. Zurek, "Pointer basis of quantum apparatus: Into what mixture does the wave packet collapse?" Physical Review D 24, 1516 (1981).

[4] W.H. Zurek, "Environment-induced superselection rules," Physical Review D 26, 1862 (1982).

[5] Eric Joos and H Dieter Zeh, "The emergence of classical properties through interaction with the environment," Zeitschrift für Physik B Condensed Matter 59, 223-243 (1985).

[6] M.A. Schlosshauer, Decoherence and the Quantum-ToClassical Transition (Springer, 2008).

[7] Oleg Lychkovskiy, "Dependence of decoherence-assisted classicality on the way a system is partitioned into subsystems," Phys. Rev. A 87, 022112 (2013).

[8] J. M. Deutsch, "Quantum statistical mechanics in a closed system," Physical Review A 43, 2046 (1991).

[9] M. Srednicki, "Chaos and quantum thermalization," Physical Review E 50, 888 (1994).

[10] M. Rigol, V. Dunjko, and M. Olshanii, "Thermalization and its mechanism for generic isolated quantum systems," Nature 452, 854-858 (2008).

[11] Takashi Mori, Tatsuhiko N Ikeda, Eriko Kaminishi, and Masahito Ueda, "Thermalization and prethermalization in isolated quantum systems: a theoretical overview," Journal of Physics B: Atomic, Molecular and Optical Physics 51, 112001 (2018)

[12] Hyungwon Kim, Tatsuhiko N. Ikeda, and David A. Huse, "Testing whether all eigenstates obey the eigenstate thermalization hypothesis," Phys. Rev. E 90, 052105 (2014)

[13] W. Beugeling, R. Moessner, and Masudul Haque, "Finitesize scaling of eigenstate thermalization," Phys. Rev. E 89, 042112 (2014)

[14] Naoto Shiraishi and Takashi Mori, "Systematic construction of counterexamples to the eigenstate thermalization hypothesis," Phys. Rev. Lett. 119, 030601 (2017).

[15] Christopher J Turner, Alexios A Michailidis, Dmitry A Abanin, Maksym Serbyn, and Zlatko Papić, "Weak ergodicity breaking from quantum many-body scars," Nature Physics 14, 745-749 (2018).
[16] Sanjay Moudgalya, Stephan Rachel, Andrei B. Bernevig, and Nicolas Regnault, "Exact excited states of nonintegrable models," Phys. Rev. B 98, 235155 (2018).

[17] Michael R. Gallis and Gordon N. Fleming, "Environmental and spontaneous localization," Phys. Rev. A 42, 38-48 (1990)

[18] L Diósi, "Quantum master equation of a particle in a gas environment," Europhysics Letters (EPL) 30, 63-68 (1995)

[19] Klaus Hornberger and John E. Sipe, "Collisional decoherence reexamined," Phys. Rev. A 68, 012105 (2003)

[20] Klaus Hornberger, "Master equation for a quantum particle in a gas," Phys. Rev. Lett. 97, 060601 (2006)

[21] Bassano Vacchini and Klaus Hornberger, "Quantum linear boltzmann equation," Physics Reports 478, 71 - 120 (2009)

[22] Walter Hahn and Boris V. Fine, "Nonentangling channels for multiple collisions of quantum wave packets," Phys. Rev. A 85, 032713 (2012)

[23] O. Lychkovskiy, "Large quantum superpositions of a nanoparticle immersed in superfluid helium," Phys. Rev. B 93, 214517 (2016).

[24] Klaus Hornberger, Stefan Uttenthaler, Björn Brezger, Lucia Hackermüller, Markus Arndt, and Anton Zeilinger, "Collisional decoherence observed in matter wave interferometry," Phys. Rev. Lett. 90, 160401 (2003).

[25] O. Lychkovskiy, "Entanglement, decoherence and thermal relaxation in exactly solvable models," Journal of Physics: Conference Series 306, 012028 (2011).

[26] Stephen M Barnett, Sonja Franke-Arnold, Aidan S Arnold, and Colin Baxter, "Coherence length for a trapped bose gas," Journal of Physics B: Atomic, Molecular and Optical Physics 33, 4177-4191 (2000)

[27] Sonja Franke-Arnold, Guillaume Huyet, and Stephen M Barnett, "Measures of coherence for trapped matter waves," J. Phys. B: At. Mol. Opt. Phys. 34, 945-964 (2001).

[28] Philip W Anderson, "Infrared catastrophe in fermi gases with local scattering potentials," Physical Review Letters 18, 1049 (1967).

[29] K. Schönhammer, "Orthogonality exponent and the friction coefficient of an electron gas," Phys. Rev. B 43, 1132311329 (1991)

[30] J. B. McGuire, "Interacting fermions in one dimension. I. Repulsive potential," J. Math. Phys. 6, 432 (1965).

[31] H Castella, X Zotos, and Peter Prelovšek, "Integrability and ideal conductance at finite temperatures," Phys. Rev. Lett. 74, 972 (1995).

[32] Oleksandr Gamayun, Oleg Lychkovskiy, and Mikhail B Zvonarev, "Zero temperature momentum distribution of an impurity in one-dimensional fermi and tonks-girardeau gases," arXiv:1909.07358 (2019).

[33] L Amico, M Boshier, G Birkl, A Minguzzi, C Miniatura, L-C Kwek, D Aghamalyan, V Ahufinger, N Andrei, AS Arnold, et al., "Roadmap on atomtronics," arXiv preprint arXiv:2008.04439 (2020).

[34] Chen Ning Yang, " $\eta$ pairing and off-diagonal long-range order in a hubbard model,' Phys. Rev. Lett. 63, 2144-2147 (1989)

[35] Daniel K Mark and Olexei I Motrunich, "Eta-pairing states as true scars in an extended hubbard model," arXiv preprint arXiv:2004.13800 (2020).

[36] Sanjay Moudgalya, Nicolas Regnault, and B Andrei Bernevig, "Eta-pairing in hubbard models: From spectrum 
generating algebras to quantum many-body scars," arXiv preprint arXiv:2004.13727 (2020).

[37] Cheng-Ju Lin, Anushya Chandran, and Olexei I. Motrunich, "Slow thermalization of exact quantum many- body scar states under perturbations," Phys. Rev. Research 2, 033044 (2020) 